\title{
Plastic surgery and specialty creep: an analysis of publication trends
}

\author{
Ethan L. Mackenzie, Jeffrey D. Larson, Samuel O. Poore \\ Division of Plastic Surgery, Department of Surgery, University of Wisconsin School of Medicine and Public Health, Madison, WI, USA
}

\begin{abstract}
Background Many surgical specialties have had pioneering influences from plastic surgeons. However, many of these areas of practice have evolved to include surgeons from diverse training backgrounds. This raises the question as to whether the prominence of other specialties in clinical practice translates to greater research productivity in these areas. The objective of this paper is to investigate the publication volumes of plastic surgeons in selected areas of practice compared to surgeons from other disciplines.

Methods PubMed was used to examine publication trends in areas associated with plastic surgery. Searches for the following topics were performed: head and neck reconstruction, hand surgery, breast reconstruction, ventral hernia repair, abdominal component separation, brachial plexus injury, craniofacial surgery, and aesthetic surgery. Affiliation tags were used to examine contributions from nine specialties. Web of Science was used to identify the top cited articles for the last 10 years in each area.

Results Articles by non-plastic surgeons comprise the majority of the literature for all areas of practice studied except for breast reconstruction and aesthetic surgery. Despite this, plastic surgeons contributed the greatest number of top cited articles over the last 10 years for five of the areas of practice.

Conclusions While plastic surgeons do not contribute the greatest proportion of articles published each year in several of the selected areas of practice, they do publish a larger number of articles that are the most cited. Plastic surgeons remain the dominant academic force in terms of volume and citations for both breast and aesthetic surgery.
\end{abstract}

Keywords Surgery, plastic / PubMed / Mammaplasty

Received: April 5, 2021 • Revised: July 21, 2021 • Accepted: August 6, 2021

pISSN: 2234-6163 • elSSN: 2234-6171 • https://doi.org/10.5999/aps.2021.00745• Arch Plast Surg 2021;48:651-659
Correspondence: Samuel 0. Poore Division of Plastic Surgery, University of Wisconsin School of Medicine and Public Health, 600 Highland Avenue, CSC G5/347, Madison, WI 53792, USA Tel: +1-608-265-7738 Fax: +1-608-265-9695 E-mail: poore@surgery.wisc.edu

\section{INTRODUCTION}

Described by some as the "last true general surgeons," plastic surgeons have a history of innovation both in and out of the operating room [1]. Historically, the roots of hand surgery, head and neck surgery, craniofacial surgery, solid organ transplantation and abdominal wall reconstruction all bear pioneering influences from plastic surgeons. Digital replants, head and neck free flaps, cleft lip repair, kidney transplants, and abdominal component separation were all introduced, at least in part, by plastic surgeons [2-6]. Despite this early history in establishing multiple subspecialties within plastic surgery, each of these fields have evolved to include surgeons from various training backgrounds including orthopedic surgeons, general surgeons, otolaryngologists, neurosurgeons and oral maxillofacial surgeons. The encroachment of other surgical specialties into areas 
of practice historically held by plastic surgeons, or "specialty creep," has taken place despite no evidence of superior outcomes by surgeons in different specialties. With plastic surgeons competing with other specialties in the clinical arena for volume in several areas of practice, it is necessary to ask whether similar competition exists with regards to contributions to the literature. In a 2016 article published by Dolan et al. [7], the authors found that non-plastic surgeons contribute robustly to the aesthetic surgery literature with regards to five specific aesthetic procedures. Unfortunately, data regarding areas of research beyond aesthetic surgery is very sparse and the literary contributions of non-plastic surgeons to the aesthetic surgery literature beyond these procedures has not been examined. To comprehensively examine the phenomenon of specialty creep in plastic surgery research, we performed a literature database study examining publishing patterns over time, including an investigation of the most influential papers that are published in specific areas of plastic surgery. Several key areas of practice were selected where non-plastic surgeons are prominent and compared the quantity and impact of publications over time to those of plastic surgeons (Table 1). Through this method of investigation, we aimed to highlight the changing role of plastic surgery in multiple areas of study.

\section{METHODS}

\section{Keyword searches}

Key phrases representing areas of plastic surgery in which there has been significant involvement of competing specialties were selected for analysis. These included "head and neck reconstruction," "hand surgery," "breast reconstruction," "ventral hernia repair," "brachial plexus surgery," "abdominal component separation," "craniofacial surgery," and "aesthetic surgery." Using these

Table 1. Key areas and non-plastic surgery specialties studied for literary contributions

\begin{tabular}{ll}
\hline Key area & $\begin{array}{c}\text { Non-plastic surgery } \\
\text { affiliations queried }\end{array}$ \\
\hline $\begin{array}{l}\text { Head and neck reconstruction } \\
\text { Hand surgery }\end{array}$ & $\begin{array}{l}\text { Otolaryngology } \\
\text { Orthopedic surgery } \\
\text { Breast reconstruction } \\
\begin{array}{l}\text { Ventral hernia and abdominal component } \\
\text { separation }\end{array}\end{array}$ \\
Brachial plexus injury & General and breast surgery \\
& Neurosurgery \\
Craniofacial surgery & Orthopedic surgery \\
Aesthetic surgery & Neurosurgery \\
& Oral maxillofacial surgery \\
& Dermatology \\
& Otolaryngology \\
\hline
\end{tabular}

key phrases, a PubMed.Gov search was conducted and the total numbers of publications were stratified by year (Supplemental Table 1). Article counts used in specialty comparisons were collected from the first year that all queried specialties had published at least one article. Using author affiliation tag searches, these were then further subdivided based on subspecialty. All searches were conducted between July 2020 and August 2020.

\section{Top cited article identification}

An additional metric of specialty influence may be seen in the author affiliations of research that is found to be the most influential in a certain specialty. To evaluate this, the most highly cited article for each field of interest over the last 10 years was also investigated by performing a Web of Science search using the above specified key phrases to search by topic. Web of Science was selected due to their search algorithm including ranking by number of times articles were subsequently cited, which is not currently available in PubMed. The most highly cited article for each key phrase per since 2010 was selected. Affiliated specialty was then determined based on listed first author affiliation tag. When such a tag was not available, affiliation was assigned based on author departmental association at the time of article publication. All articles were reviewed and articles that were unrelated to the search topic were excluded (Supplemental Table 2).

\section{Statistical analyses}

All data obtained from both PubMed and Web of Science was downloaded into spreadsheet software (Excel; Microsoft Corp., Redmond, WA, USA). The frequency and proportion of publications for each key phrase were calculated using in-program statistical functions. Year-on-year percent change in publication was also determined. Descriptive statistics and Student t-test or analysis of variance (ANOVA) were calculated for publications by specialty over 3-year periods using Microsoft Excel. Microsoft Excel was used for graphical representations of data.

\section{RESULTS}

\section{Head and neck reconstruction}

A total of 17,164 articles were identified from 1987 to 2019 . There was a drastic increase in total number of publications produced with only 62 articles published in 1987 compared to 1,458 in 2019. Similarly, an accompanying increase in publications was seen among both otolaryngology and plastic surgery specialties for head and neck reconstruction (Fig. 1A). During the entire period studied, more head and neck reconstructive papers were published by authors with otolaryngology affiliations (Fig. 1, Supplemental Table 3). However, during three spe- 

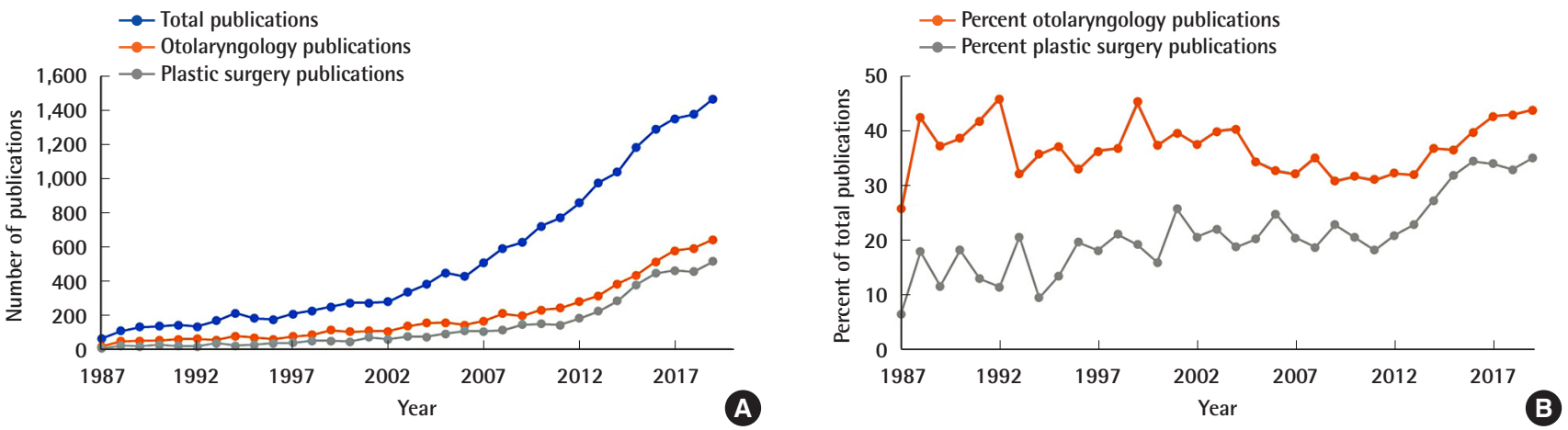

Fig. 1. Head and neck reconstruction (A) publications per year and (B) percent of total publications per year for otolaryngology and plastic surgery.
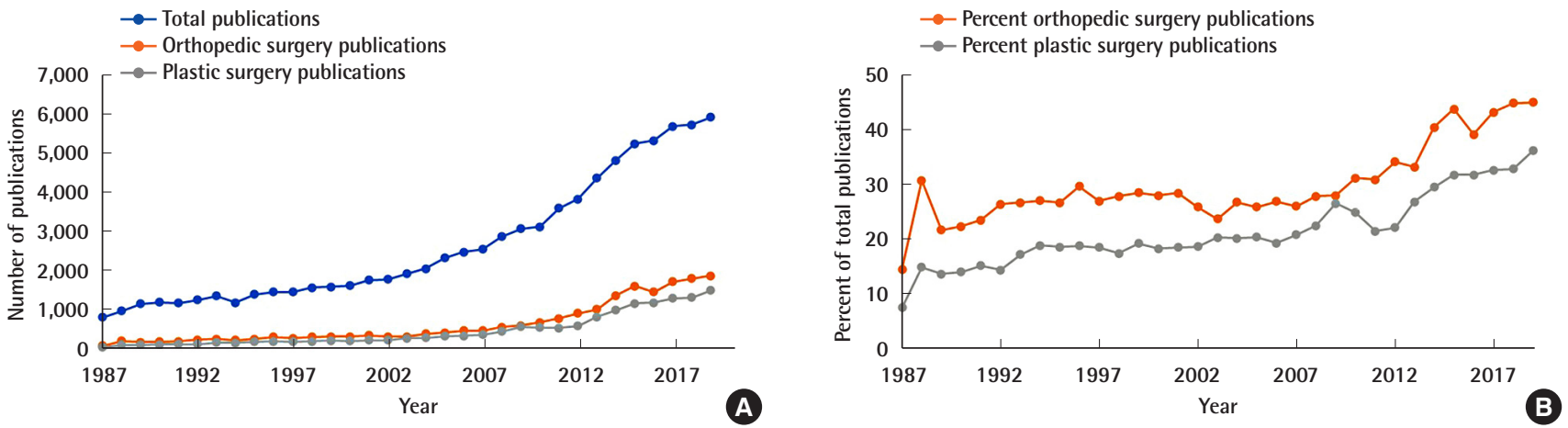

Fig. 2. Hand surgery (A) publications per year and (B) percent of total publications per year for orthopedic surgery and plastic surgery.

cific time periods (1987-1989, 2011-2013, and 2014-2016), there was no significant difference between the number of publications with otolaryngology author affiliations as compared to plastic surgeon author affiliations $(\mathrm{P}>0.05)$.

\section{Hand surgery}

A total of 87,152 articles were identified from 1987 to 2019 . The available hand literature dates back over 150 years with the oldest article found dating from 1868 . The number of articles published with both plastic surgery and orthopedic surgery authors increased from 43 and 83 respectively in 1987 to 1,509 and 1,878 respectively in 2019 (Fig. 2A). During the entire period studied, more hand surgery papers were published by authors with orthopedic surgery affiliations, comprising a greater proportion of the total literature (Fig. 2). However, during the 2008-2010 and 2011-2013 time periods, there was no significant difference between number of publications with either author affiliation $(P>0.05)$ with orthopedic surgery publishing significantly more for all other time periods (Supplemental Table 4).

\section{Breast reconstruction}

A total of 20,567 articles were identified from the study date range. The number of articles published with both plastic surgery and general/breast surgery authors increased from 12 and 3 re- spectively in 1987 to 851 and 175 respectively in 2019 (Fig. 3A). During the entire period studied, more breast reconstruction papers were published by authors with plastic surgery affiliations, comprising a greater proportion of the total literature (Fig. 3). This difference was significant for all time periods following 1995 (P > 0.05) (Supplemental Table 5). Since 2015, plastic surgery has accounted for greater than $50 \%$ of published breast reconstruction literature (Fig. 3B).

\section{Ventral hernia}

A total of 8,851 articles were identified from 1988 to 2019 with the term "ventral hernia." While articles published by general surgeons have increased substantially from 1988 to 2019, those published by plastic surgeons have increased much more modestly from two articles to 26 articles in 1988 and 2019 respectively (Fig. 4A). During the entire period studied, more ventral hernia papers were published by authors with general surgery affiliations, comprising a greater proportion of the total literature (Fig. 4). In order to eliminate areas of the literature without expected plastic surgery contributions, such as laparoscopic hernia repair, a refined search with the term "abdominal component separation" was conducted. Proportions of published articles were much more similar for this search term (Fig. 5). For a period from 1990, the first year that had identified articles pub- 

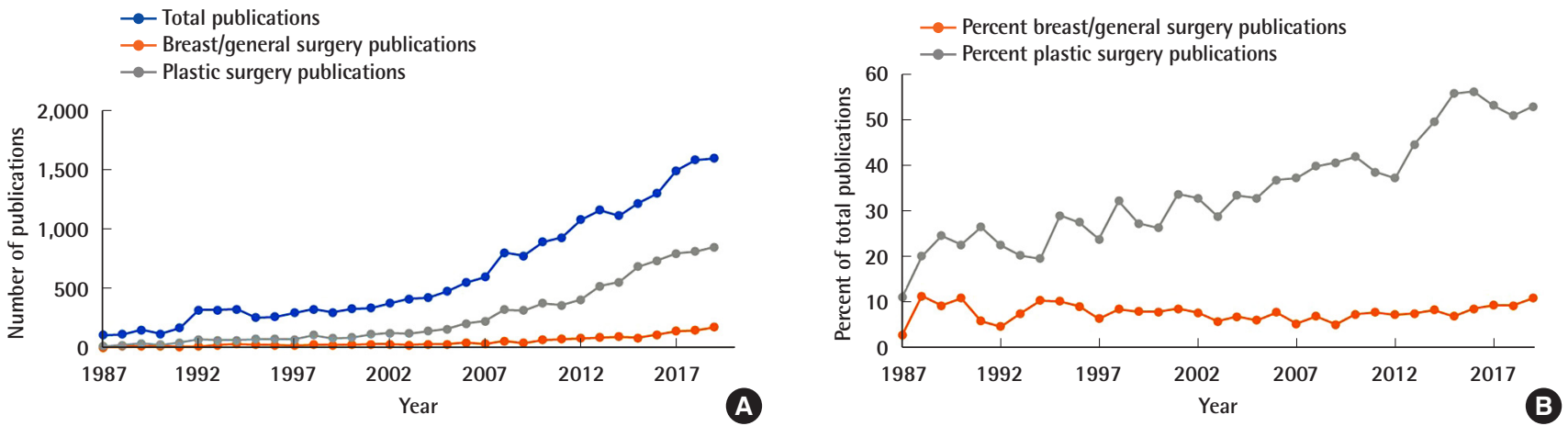

Fig. 3. Breast reconstruction (A) publications per year and (B) percent of total publications per year for breast/general surgery and plastic surgery.
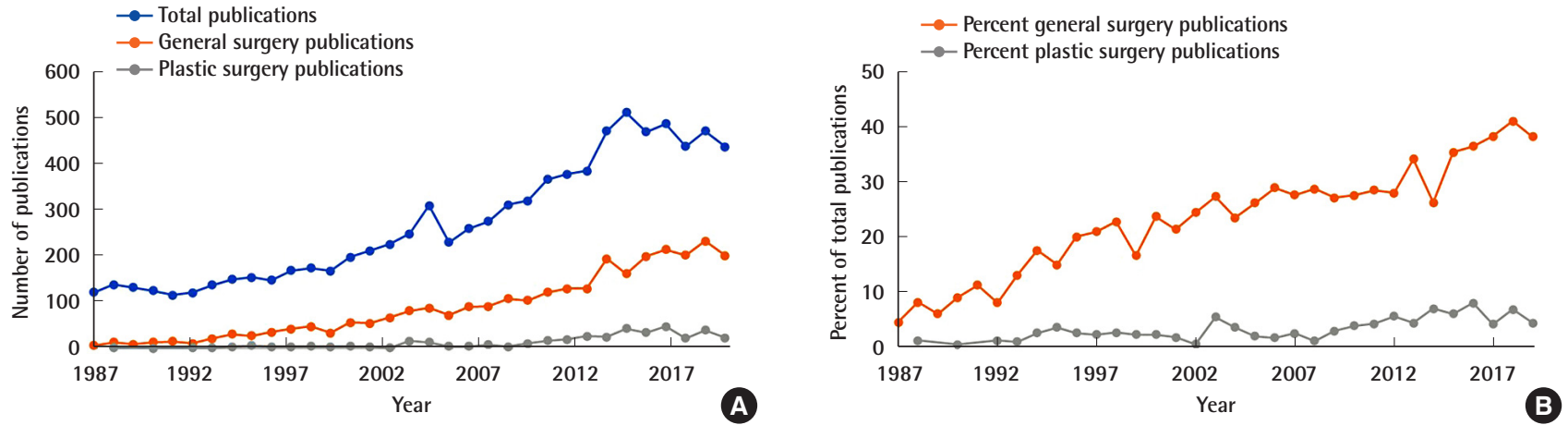

Fig. 4. Ventral hernia (A) publications per year and (B) percent of total publications per year for general surgery and plastic surgery.
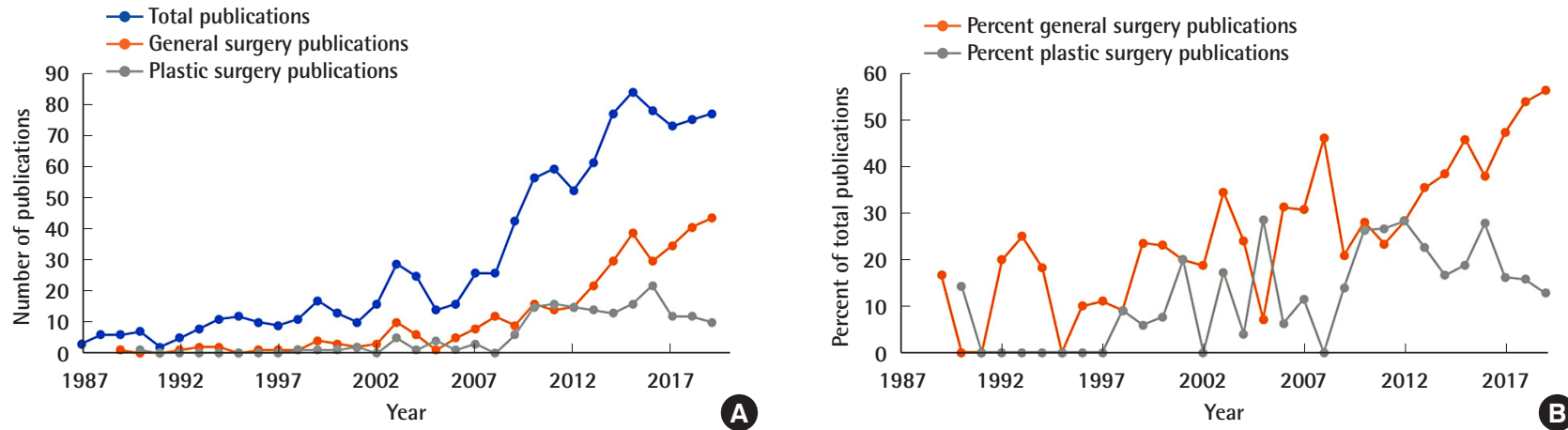

Fig. 5. Abdominal component separation (A) publications per year and (B) percent of total publications per year for general surgery and plastic surgery.

lished by both general surgeons and plastic surgeons, to 2013 there was no significant differences in the number of articles published by either specialty. However, since 2013, general surgeons have published significantly more under this search term (Supplemental Table 6).

\section{Brachial plexus injury}

A total of 11,042 articles were identified from 1987 to 2019 . Neurosurgery and plastic surgery contributions to the literature have comprised approximately $10 \%$ each of the literature over the last several years. The orthopedic surgery literature has comprised the largest plurality of author affiliations during the entire period studied, with this proportion increasing over the last 10 years (Fig. 6). During the entire study period, ANOVA found significant differences between the groups studied $(\mathrm{P}<0.05)$ (Supplemental Table 7).

\section{Craniofacial surgery}

A total of 15,318 articles were identified from 1987 to 2019 . Since 2010, oromaxillofacial surgery (OMFS) has published the largest plurality of articles. Prior to 2010, OMFS and plastic surgery contributions represented relatively similar proportions of the literature with neurosurgery comprising significantly less (Fig. 7). During the entire study period, from 1989 to 2019, 

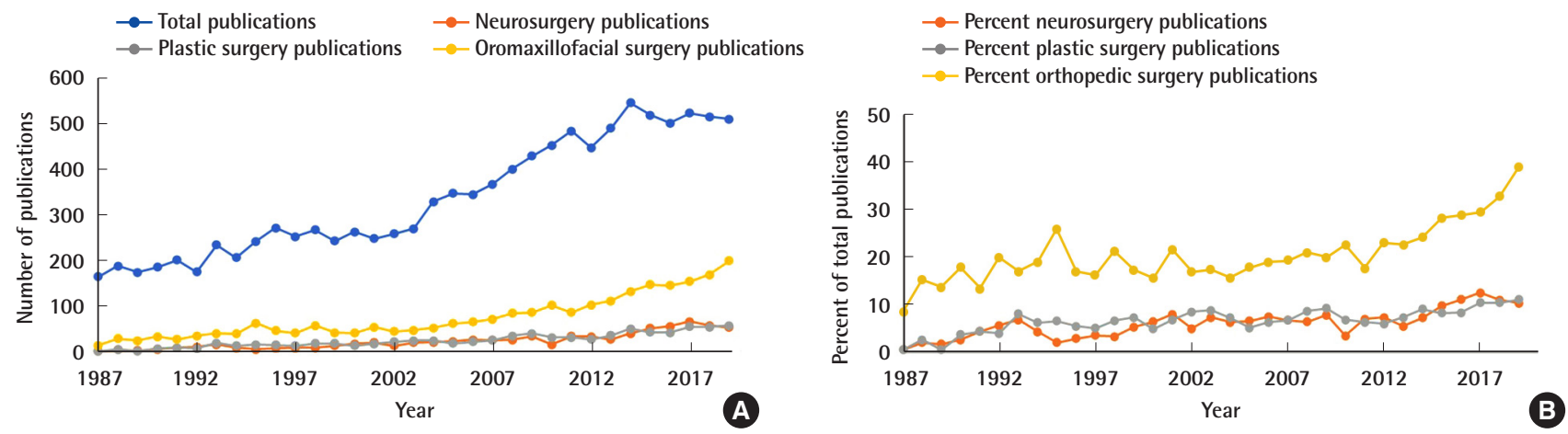

Fig. 6. Brachial plexus injury (A) publications per year and (B) percent of total publications per year for neurosurgery, orthopedic surgery, and plastic surgery.
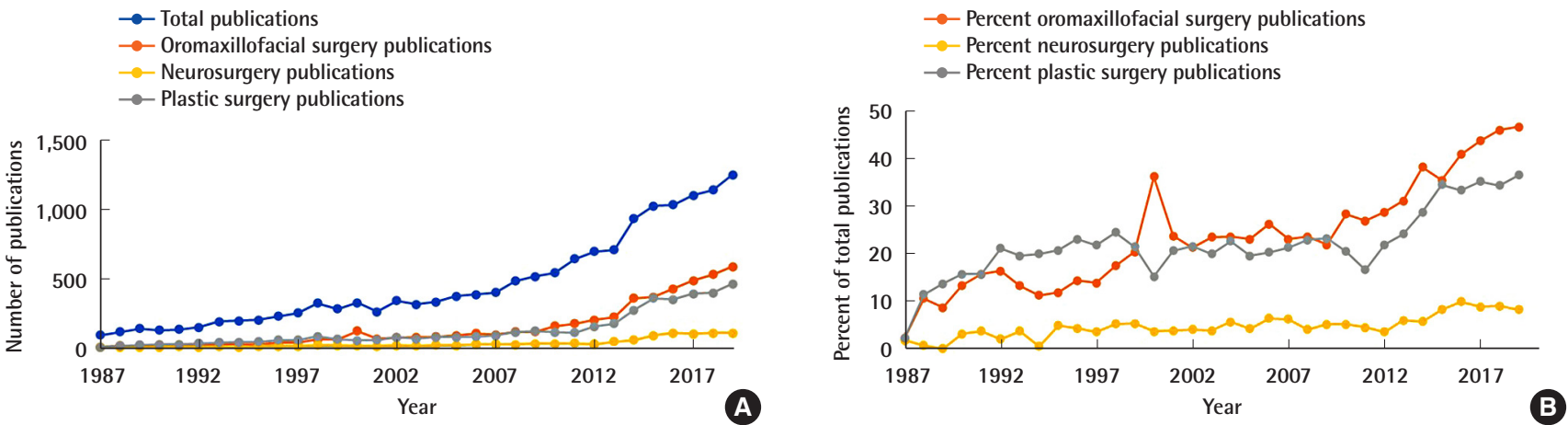

Fig. 7. Craniofacial surgery (A) publications per year and (B) percent of total publications per year for oromaxillofacial surgery, neurosurgery, and plastic surgery.
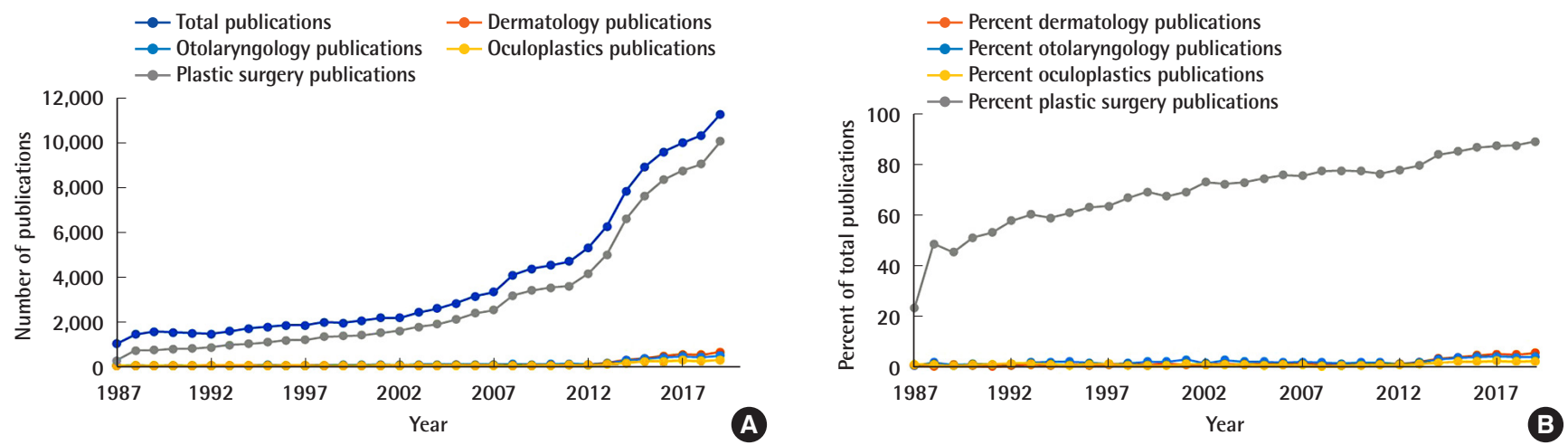

Fig. 8. Aesthetic surgery (A) publications per year and (B) percent of total publications per year for dermatology, otolaryngology, oculoplastics, and plastic surgery.

ANOVA found significant differences between the groups studied $(\mathrm{P}<0.05)$ (Supplemental Table 8).

\section{Aesthetic surgery}

A total of 128,077 articles were identified from 1987 to 2019 . Overall number of publications have increased significantly over time with 982 articles published in 1987 compared to 12,537 in 2019. While publication numbers have increased for all specialties studied, otolaryngology, dermatology, oculoplastic surgery, and plastic surgery, a large majority of publications are pub- lished by authors with plastic surgery affiliations throughout this time period (Fig. 8, Supplemental Table 9). Analysis of variance testing shows differences in publishing volumes between the specialties to be significant over the entire period studied $(\mathrm{P}<0.05)$.

\section{Top cited articles}

In terms of top cited publications, there are several areas of practice in which plastic surgeons published a greater plurality of top cited publications over the last 10 years (Table 2). For head and 
Table 2. Specialty affiliation of the first author of top cited article published between 2010 and 2019 for each key practice area

\begin{tabular}{|c|c|c|c|c|c|c|c|}
\hline Year & Head and neck & Hand surgery & Breast reconstruction & Ventral hernia & Brachial plexus injury & Craniofacial surgery & Aesthetic surgery \\
\hline 2019 & ENT & Orthopedic surgery & Plastic surgery & General surgery & Neurosurgery & Anesthesia & Plastic surgery \\
\hline 2018 & OMFS & Orthopedic surgery & Radiation oncology & General surgery & Plastic surgery & Plastic surgery & Plastic surgery \\
\hline 2017 & ENT & Plastic surgery & Plastic surgery & Anesthesia & Neurosurgery & Plastic surgery & Plastic surgery \\
\hline 2016 & Plastic surgery & Plastic surgery & Plastic surgery & General surgery & $\begin{array}{l}\text { Obstetrics and } \\
\text { gynecology }\end{array}$ & Plastic surgery & Infectious disease \\
\hline 2015 & OMFS & Plastic surgery & Breast surgery & General surgery & Plastic surgery & Plastic surgery & Plastic surgery \\
\hline 2014 & Plastic surgery & Rheumatology & Radiation oncology & General surgery & $\begin{array}{l}\text { Obstetrics and } \\
\text { gynecology }\end{array}$ & Plastic surgery & Plastic surgery \\
\hline 2013 & ENT & Plastic surgery & Plastic surgery & General surgery & Biology & Plastic surgery & Plastic surgery \\
\hline 2012 & Plastic surgery & Orthopedic surgery & Plastic surgery & General surgery & Neurosurgery & Laboratory medicine & Plastic surgery \\
\hline 2011 & Plastic surgery & Plastic surgery & Plastic surgery & General surgery & Orthopedic surgery & Anesthesia & $\begin{array}{c}\text { Obstetrics and } \\
\text { gynecology }\end{array}$ \\
\hline 2010 & Plastic surgery & Anesthesia & Plastic surgery & Plastic surgery & Orthopedic surgery & Molecular medicine & Plastic surgery \\
\hline
\end{tabular}

OMFS, oromaxillofacial surgery; ENT, otolaryngology.

neck reconstruction, the top cited article for 3 of the last 10 years was published with otolaryngology author affiliations as compared to five for plastic surgery (Supplemental Table 10). The remaining two were published by oral maxillofacial surgeons. For hand surgery, similarly, three of the top cited articles were published by orthopedic surgeons as compared to five for plastic surgery and two published by specialists in anesthesiology or rheumatology (Supplemental Table 11). In breast reconstruction, an area of practice traditionally dominated by plastic surgeons, the top cited article for 7 of the last 10 years was published with plastic surgery author affiliations as compared to one for breast/general surgery (Supplemental Table 7). The remaining two were published by radiation oncologists although there were non-first author plastic surgeons on these publications (Supplemental Table 12). Conversely, for ventral hernia the top cited article for 8 of the last 10 years was published with general surgery author affiliations as compared to one for plastic surgery (Supplemental Table 13). The remaining paper was published by specialists in the field of anesthesiology. With regards to brachial plexus injury, the top cited article for 3 of the last 10 years was published with neurosurgery author affiliations as compared to two for plastic surgery and two for orthopedic surgery (Supplemental Table 14). In terms of craniofacial surgery, the top cited article for 6 of the last 10 years was published with plastic surgery author affiliations as compared to none for either OMFS or neurosurgery. The contributions for the remaining 4 years were made by anesthesiologists or basic scientists without primary surgical affiliations (Supplemental Table 15). Lastly, plastic surgeons strongly contribute to the top cited articles for aesthetic surgery with 8 of the last 10 years was published with plastic surgery author affiliations with the other two published by authors with infectious disease or obstetrics and gynecology affiliations (Supplemental Table 16).

\section{DISCUSSION}

Our study demonstrates that plastic surgeons share publishing space with several competing specialties including dermatology, oculoplastic surgery, orthopedic surgery, general surgery, otolaryngology, neurosurgery, and oral maxillofacial surgery. While plastic surgery maintains a publishing presence in multiple areas of practice, several have become increasingly dominated by competing surgical specialties. However, plastic surgeons have maintained a robust publishing presence in several areas, most convincingly aesthetic surgery and breast reconstruction. Furthermore, publication trends in all areas studied have largely demonstrated year-on-year increases in publishing volumes. Additionally, even in fields where plastic surgery is outperformed in publishing volume by other surgical disciplines, a large proportion of top cited articles are still published by plastic surgeons with this assertion holding true over the course of the last 10 years. This is perhaps best seen in craniofacial surgery and head and neck reconstruction where plastic surgeons published the largest plurality of top papers over the time period studied.

Head and neck reconstruction is an area which has become increasingly dominated by otolaryngology. Based on data from the American College of Surgeons National Surgical Quality Improvement Program, between 2005 and 2015, otolaryngologists performed 1,429 free tissue transfers for head and neck reconstruction compared to 893 performed by plastic surgeons [8]. In spite of this, flap outcomes appear to be either comparable or superior for plastic surgeons as compared to otolaryngologists $[8,9]$. Beyond the operating room, over the period studied, otolaryngology publications on head and neck reconstruction outnumbered those published by plastic surgeons every year. Interestingly, the divide between the volume published by each specialty appears to have narrowed over the time of the study 
period, perhaps suggestive of increasing plastic surgery contribution to research in this field. Additionally, despite publishing fewer papers on head and neck reconstruction, plastic surgeons published the top most cited article for 3 of the last 10 years compared to three articles published by otolaryngologists.

Despite some fluctuation, the difference between volume of hand surgery publications written by orthopedic surgeons and those by plastic surgeons has remained relatively constant with orthopedic surgeons consistently publishing more. In spite of these lower publishing volumes, plastic surgeons again published a greater number of top-cited articles (five compared to three). There has been significant concern raised in recent years over the diminishing presence of plastic surgeons. High volume hand fellowships such as the Curtis Hand Center have seen significantly more fellows of orthopedic background graduate recently as compared to fellows with primary plastic surgery training [10]. This is reflected in national trends; 204 hand fellows with a primary plastic surgery background graduated between 2000 and 2010 compared with 785 hand fellows from a primary orthopedic surgery background [11]. Although orthopedic surgeons publish a larger proportion of the hand surgery literature, plastic surgery contributions have kept pace and have had an overall larger impact based on citations.

One area that has remained primarily under the realm of plastic surgery is breast reconstruction. An exception to this is the area of oncoplastic breast reduction in which a majority of oncoplastic procedures are performed by general/breast surgeons with no difference in complication rates [12]. The proportion of breast reconstruction papers published by plastic surgeons has grown over time to over $50 \%$ of the literature published each year. In recent years, the proportion of articles published by breast and general surgeons has also grown, perhaps reflective of increasing surgical contributions. However, with the top cited article for 7 of the last 10 years, plastic surgeons remain both academically and clinically dominant in breast reconstruction.

The modern component separation technique for closure of ventral hernia defects was pioneered by Ramirez et al. [3], a plastic surgeon. However, over the past 30 years, ventral hernia repair has largely become the domain of general surgeons. At least some of this is likely due to advances in laparoscopic technique and increasing prevalence of laparoscopic ventral hernia repairs. Accompanying this, publications addressing ventral hernias have been overwhelmingly published by general surgeons. This is also seen with authorship patterns of the top cited articles. However, when focusing specifically on abdominal component separation, it is only in recent years that general surgeons have published larger volumes than plastic surgeons and since 2014, this difference has only increased. As such, it appears that this is one field in which plastic surgeons have seen diminished influence in the primary literature.

Injury of the brachial plexus often requires multidisciplinary approaches and multiple specialties provide important contributions to the literature. While both plastic surgeons and neurosurgeons provide modest contributions to the literature that have grown at approximately the same rate, neither approach the volume of literature contributed by orthopedic surgeons. Despite this, each of the three specialties studied appear to have contributed approximately equal numbers of top cited articles over the last 10 years. Given the collaborative nature of care for brachial plexus injuries, plastic surgery is likely to maintain an academically significant, albeit modest, contribution to the literature.

Craniofacial surgery is a vast area of subspecialization ranging from care for traumatic injuries to correction of congenital abnormalities of both the soft tissue and skeletal structures of the face and cranium. Both plastic surgeons and OMFS surgeons contribute similar volume of publications with substantially less contributed by neurosurgeons. Despite OMFS surgeons contributing slightly more in recent years, plastic surgeons contributed six of the top cited articles over the last 10 years demonstrating both a robust and impactful literary contribution.

Aesthetic surgery is perhaps the most public facing aspect of plastic surgery. A proliferation of non-plastic surgeons offering aesthetic procedures has seen increasing marketplace competition between specialties $[13,14]$. This competition occurs at a time when a significant proportion graduating plastic surgery residents feel uncomfortable performing certain cosmetic surgery procedures without pursuing further training [15]. Previously published analyses of aesthetic surgery publication trends have found that plastic surgeons maintain a strong publication presence with regards to selected areas of the aesthetic surgery literature $[7,16]$. Our analysis concurs with these findings, demonstrating that authors with plastic surgery affiliations are responsible for an overwhelming majority of the aesthetic surgery literature, however with specialties such as obstetrics and gynecology responsible for highly cited papers, plastic surgeons must continue to cultivate research in aesthetic surgery to remain leaders in this field.

This study has several limitations based on research methodology. The number of publications analyzed is directly influenced by the Pubmed.Gov search syntax utilized and while searches were designed to target as many relevant papers as possible, it is always possible that some proportion were missed. Additionally, rather than using medical subject heading $(\mathrm{MeSH})$ terms, keywords were used in searches. This is due to the fact that $\mathrm{MeSH}$ terms do not have the required specificity for many of the subjects studied here and do not allow a similar level of flexibility as 
keywords for search refinement. Furthermore, the number of articles published in a practice area by a particular specialty is likely to be, in part, a function of the total number of surgeons practicing in the field as well as the academic productivity of individual members of those specialties. However, performing a "per capita" adjustment would be difficult to do without specific data regarding the number of actively practicing surgeons in each specialty.

In selecting for all papers returned by a search with a specific keyword, there is potential for confoundment of results based on regional differences. For instance, inclusion of breast reconstructive papers published by European surgeons, where much breast reconstruction has been traditionally performed by breast surgeons, may affect the perception of publishing trends in the United States where the majority of breast reconstruction is performed by plastic surgeons. Furthermore, our analysis of the various practice areas does not parse the data to the level of specific procedures.

While we endeavored to include a wide variety of areas of practice in which plastic surgeons contribute to the literature, the list chosen here is by no means comprehensive. The areas selected were chosen based on their prominence within the field of plastic surgery including several with defined fellowships (hand and peripheral nerve, craniofacial, aesthetic, microsurgery for both breast and head and neck reconstructions). However, the breadth of the field of practice of plastic surgery means that there were many areas not included here. Additionally, there may be surgical specialties which also practice in these areas which were not included for brevity purposes.

Plastic surgery has perhaps the widest breadth of any surgical subspecialty, operating all over the human body. This broad clinical purview, along with a strong history of innovation, means plastic surgeons have pioneering influences in many areas of clinical practice. However, due to clinical overlap with other subspecialties, many of these clinical areas have been adopted by other surgical specialties that continue to both develop surgical technique and publish in these areas. Despite sharing intellectual space with a multitude of other specialties, plastic surgery maintains a robust publishing presence and appears to have an outsize influence on the literature as compared to other subspecialties. These findings are perhaps most obvious within aesthetic surgery, however with the increasing presence of other specialties in the clinical practice of aesthetic surgery, we must strive to continue to innovate and publish, marrying research with clinical practice to provide superior results to patients.

\section{NOTES}

\section{Conflict of interest}

No potential conflict of interest relevant to this article was reported.

\section{Author contribution}

Conceptualization: EL MacKenzie, SO Poore. Data curation: EL MacKenzie, JD Larson, SO Poore. Formal analysis: EL MacKenzie. Methodology: EL MacKenzie, JD Larson, SO Poore. Project administration: JD Larson, SO Poore. Visualization: EL MacKenzie. Writing - original draft: EL MacKenzie. Writing - review \& editing: EL MacKenzie, JD Larson, SO Poore.

\section{ORCID}

Ethan L. Mackenzie https://orcid.org/0000-0002-8857-7888

Jeffrey D. Larson https://orcid.org/0000-0002-9518-5129

Samuel O. Poore https://orcid.org/0000-0002-6029-3306

\section{Supplementary material}

Supplemental Table 1. PubMed search syntax. Supplemental data can be found at: https://doi.org/10.5999/aps.2021.00745

Supplemental Table 2. Article exclusions from most cited analysis. Supplemental data can be found at: https://doi.org/10.5999/ aps.2021.00745

Supplemental Table 3. Descriptive statistics and two-way t-tests for head and neck reconstruction publications by 3 year periods comparing otolaryngology and plastic and reconstructive surgery. Supplemental data can be found at: https://doi.org/10.5999/ aps.2021.00745

Supplemental Table 4. Descriptive statistics and two-way t-tests for hand surgery publications by 3 year periods comparing orthopedic surgery and plastic and reconstructive surgery. Supplemental data can be found at: https://doi.org/10.5999/aps. 2021.00745

Supplemental Table 5. Descriptive statistics and two-way t-tests for breast reconstruction publications by 3 year periods comparing breast/general surgery and plastic and reconstructive surgery. Supplemental data can be found at: https://doi.org/10.5999/ aps.2021.00745

Supplemental Table 6. Descriptive statistics and two-way t-tests for abdominal component separation publications by 3 year periods comparing general surgery and plastic and reconstructive 
surgery. Supplemental data can be found at: https://doi.org/ 10.5999/aps.2021.00745

Supplemental Table 7. Descriptive statistics and ANOVA for brachial plexus injury publications by 3 year periods comparing neurosurgery, orthopedic surgery, and plastic and reconstructive surgery. Supplemental data can be found at: https://doi. org/10.5999/aps.2021.00745

Supplemental Table 8. Descriptive statistics and ANOVA for craniofacial surgery publications by 3 year periods comparing oromaxillofacial surgery, neurosurgery, and plastic and reconstructive surgery. Supplemental data can be found at: https:// doi.org/10.5999/aps.2021.00745

Supplemental Table 9. Descriptive statistics and ANOVA for aesthetic surgery publications by 3 year periods comparing dermatology, otolaryngology, oculoplastics, and plastic and reconstructive surgery. Supplemental data can be found at: https:// doi.org/10.5999/aps.2021.00745

Supplemental Table 10. The top cited article by year from 2010 to 2019 for head and neck reconstruction. Supplemental data can be found at: https://doi.org/10.5999/aps.2021.00745

Supplemental Table 11. The top cited article by year from 2010 to 2019 for hand surgery. Supplemental data can be found at: https://doi.org/10.5999/aps.2021.00745.

Supplemental Table 12. The top cited article by year from 2010 to 2019 for breast reconstruction. Supplemental data can be found at: https://doi.org/10.5999/aps.2021.00745

Supplemental Table 13. The top cited article by year from 2010 to 2019 for ventral hernia. Supplemental data can be found at: https://doi.org/10.5999/aps.2021.00745

Supplemental Table 14. The top cited article by year from 2010 to 2019 for brachial plexus injury. Supplemental data can be found at: https://doi.org/10.5999/aps.2021.00745

Supplemental Table 15 . The top cited article by year from 2010 to 2019 for craniofacial surgery. Supplemental data can be found at: https://doi.org/10.5999/aps.2021.00745

Supplemental Table 16. The top cited article by year from 2010 to 2019 for aesthetic surgery. Supplemental data can be found at: https://doi.org/10.5999/aps.2021.00745

\section{REFERENCES}

1. Neligan PC. Plastic surgery. London: Elsevier Saunders; 2013.

2. Barry JM, Murray JE. The first human renal transplants. J Urol 2006; 176:888-90.

3. Ramirez OM, Ruas E, Dellon AL. "Components separation" method for closure of abdominal-wall defects: an anatomic and clinical study. Plast Reconstr Surg 1990;86:519-26.

4. Buncke HJ, Alpert BS, Johnson-Giebink R. Digital replantation. Surg Clin North Am 1981;61:383-94.

5. Daniel RK, Taylor GI. Distant transfer of an island flap by microvascular anastomoses: a clinical technique. Plast Reconstr Surg 1973;52:111-7.

6. Millard DR Jr. Refinements in rotation-advancement cleft lip technique. Plast Reconstr Surg 1964;33:26-38.

7. Dolan RT, Zins JE, Morrison CM. The aesthetic surgery literature: do plastic surgeons remain at the cutting edge? Plast Reconstr Surg 2016;138:277-87.

8. Drinane JJ, Drinane J, Nair L, et al. Head and neck reconstruction: does surgical specialty affect complication rates? J Reconstr Microsurg 2019;35:516-21.

9. Kordahi AM, Hoppe IC, Lee ES. A comparison of free tissue transfers to the head and neck performed by sugeons and otolaryngologists. J Craniofac Surg 2016;27:e82-5.

10. Higgins JP. The diminishing presence of plastic surgeons in hand surgery: a critical analysis. Plast Reconstr Surg 2010; 125:248-60.

11. Goldfarb CA, Lee WP, Briskey D, et al. An American Society for Surgery of the Hand (ASSH) task force report on hand surgery subspecialty certification and ASSH membership. J Hand Surg Am 2014;39:330-4.

12. Blankensteijn LL, Crystal DT, Egeler SA, et al. The influence of surgical specialty on oncoplastic breast reconstruction. Plast Reconstr Surg Glob Open 2019;7:e2248.

13. Housman TS, Hancox JG, Mir MR, et al. What specialties perform the most common outpatient cosmetic procedures in the United States? Dermatol Surg 2008;34:1-8.

14. D’Amico RA, Saltz R, Rohrich RJ, et al. Risks and opportunities for plastic surgeons in a widening cosmetic medicine market: future demand, consumer preferences, and trends in practitioners' services. Plast Reconstr Surg 2008;121:1787-92.

15. McNichols CH, Diaconu S, Alfadil S, et al. Cosmetic surgery training in plastic surgery residency programs. Plast Reconstr Surg Glob Open 2017;5:e1491.

16. Waltzman JT, Tadisina KK, Orra S, et al. Who is publishing in facial cosmetic surgery? A citation analysis across specialties over five decades. Aesthet Surg J 2016;36:743-55. 